Published in final edited form as:

South Med J. 1977 April ; 70(4): 389-390.

\title{
CURRENT STATUS OF LIVER TRANSPLANTATION
}

\author{
Thomas E. Starzl, MD, Charles W. Putnam, MD, and Lawrence J. Koep, MD \\ Department of Surgery, University of Colorado Medical Center, 4200 E Ninth Ave, Denver, Colo \\ 80262
}

Liver replacement with a homograft (orthotopic liver transplantation) was first attempted in a human on March 1, 1963. ${ }^{1}$ Since then more than a hundred patients have been treated with this procedure at the University of Colorado. More than a hundred additional cases have been accumulated in other centers. The second most active group in the field has been composed of personnel at Addenbrooke Hospital, Cambridge, and at King's College Hospital, London. The British inter-university effort has involved the fruitful collaboration of the surgeon, Professor Roy Calne, and the gastroenterologist, Professor Roger Williams, and has resulted in approximately 50 orthotopic liver transplantations.

In the 13 years of our own experience, the most meaningful conclusion has been that prolonged survival was repeatedly possible. ${ }^{2}$ Among our first 100 recipients, 28 lived at least one postoperative year, 15 are still alive, and the longest follow-up is now 6.6 years. This last patient was a 3-year-old-child with biliary atresia. Three other recipients in our series have lived more than five years after transplantation, two for Wilson's disease and another for biliary atresia.

Most of the long-term survivors, particularly those in the last several years, have had stable liver function and thus have been able to achieve social and vocational rehabilitation equal to that of kidney homograft recipients. ${ }^{2}$ Long-term survival after liver transplantation has also been achieved by teams at Cambridge-King's College, Commonwealth of Virginia, University of Montreal, and New York Memorial Hospital.

Conceding that the feasibility stage of liver transplantation has long been passed, the important issues now being examined are the reasons why the one-year mortality has remained high and how this situation can be improved. One explanation is that the operation can be extraordinarily difficult. In children we have encountered a very high incidence of technical complications including homograft ischemic necrosis, graft vascular thrombosis, uncontrolled hemorrhage, and, above all, biliary duct complications. ${ }^{3}$ All of these misadventures, except for biliary duct problems, tend to cause death within the first few days after operation.

Liver transplantation in adults can be even more formidable because of the nearly uncontrollable hemorrhage caused by a combination of deficient clotting factors and severe portal hypertension, which arises during operation in patients with end-stage liver disease. Adults, no less than children, have suffered a high mortality from biliary fistulas and obstructions. Finally, irreversible brain injury during or just after operation has cost the life of about one fifth of our adult recipients. Our suspicion is that much of the difficulty was caused by air entrapped in the graft which then embolized in tiny bubbles through the pulmonary bed into the systemic circulation.

As the foregoing technical and management problems have become better defined, preventive measures have evolved. The use of microsurgical technics has reduced vascular problems in pediatric recipients. Biliary reconstruction and the treatment of biliary complications have greatly improved in the last two or three years. The threat of air 
embolism can be markedly reduced by slowly perfusing the homograft portal vein with an electrolyte solution during performance of the vascular anastomoses, thus filling the vascular tree.

Correction of these various faults should lead to a favored position of the liver among organ grafts. In rats, dogs, and pigs, the liver is not as vigorously rejected as the kidney and other tissues. Rejection has been an uncommon feature in the livers examined at autopsies of our unsuccessfully treated liver recipients. ${ }^{2}$ Far more commonly, serious mechanical imperfections have been found.

As progress of the kind already described continues, liver transplantation will be more freely applied at an earlier time. We presently believe recipients should be young, 45 years or less. The ideal indication for liver replacement is otherwise untreatable, chronic, non-neoplastic hepatic disease, such as biliary atresia or chronic aggressive hepatitis. Although our experience has been poor in Laennec's cirrhosis, the inferior results probably reflect, for the most part, excessively late consideration of transplantation. Inborn errors of metabolism so far "cured" by liver replacement include Wilson's disease, alpha ${ }_{1}$-antitrypsin deficiency, Niemann-Pick disease, and tyrosinemia.

The role of liver transplantation for primary hepatic malignancy will have to be clarified in future trials. It is clear that a very high rate of recurrence can be expected in hepatomas, cholangiocarcinomas, intrahepatic duct cell carcinomas, and less common malignant neoplasms. Yet, we now have two patients living, one after two years and the other after six years, following whole liver resection for an intrahepatic duct cell carcinoma and for a hepatoma, respectively.

At the moment, liver transplantation remains experimental, but with the proviso that many patients have benefited from the procedure. There is little reason to doubt that hepatic replacement will be a more common operation in the years to come. A shortage of organs may then present an obstacle, as has occurred with the kidney. The resulting logistic problems of organ storage and shipment are solvable, however. Using simple infusion with cold Collins' solution, we recently removed a liver in Los Angeles, carried it to Denver on a commercial airliner, and successfully transplanted it after an ischemic period of seven hours.

\section{Acknowledgments}

This work was supported by research projects MRIS 8118-01 and 7227-01 from the Veterans Administration; by Grants AM-17260 and AM-07772 from the National Institutes of Health; and by Grants RR-00051 and RR-00069 from the General Clinical Research Centers Program of the Division of Research Resources, National Institutes of Health.

\section{References}

1. Starzl, TE.; Putnam, CW. Experience in Hepatic Transplantation. Philadelphia: W.B. Saunders Co.; 1969.

2. Starzl TE, Porter KA, Putnam CW, et al. Orthotopic liver transplantation in 93 patients. Surg Gynecol Obstet. 1976; 142:487-505. [PubMed: 176741]

3. Starzl TE, Porter KA, Putnam CW, et al. Biliary complications after liver transplantation: with special reference to the biliary cast syndrome and techniques of secondary duct repair. Surgery. (In press). 